\title{
Gradhiva
}

GRADHIV

Revue d'anthropologie et d'histoire des arts

33 | 2022

Wampum : les perles de la diplomatie

\section{Un collier pour les lier, un collier pour les trouver, un collier pour les ramener tous chez eux}

A belt to bind them, a belt to find them, a belt to bring them all back home

\section{Nikolaus Stolle}

Traducteur : Lucie Modde

\section{(2) OpenEdition \\ Journals}

Édition électronique

URL : https://journals.openedition.org/gradhiva/5990

DOI : 10.4000/gradhiva.5990

ISSN : $1760-849 x$

Cet article est une traduction de :

A Belt to Bind Them, to Find Them, to Bring Them all Back Home - URL : https://

journals.openedition.org/gradhiva/6205 [en]

Éditeur

Musée du quai Branly Jacques Chirac

Édition imprimée

Date de publication : 2 février 2022

Pagination : 60-77

ISBN : 978-2-35744-133-0

ISSN : 0764-8928

Référence électronique

Nikolaus Stolle, « Un collier pour les lier, un collier pour les trouver, un collier pour les ramener tous chez eux », Gradhiva [En ligne], 33 | 2022, mis en ligne le 02 février 2022, consulté le 14 septembre 2022. URL : http://journals.openedition.org/gradhiva/5990 ; DOI : https://doi.org/10.4000/gradhiva. 5990 


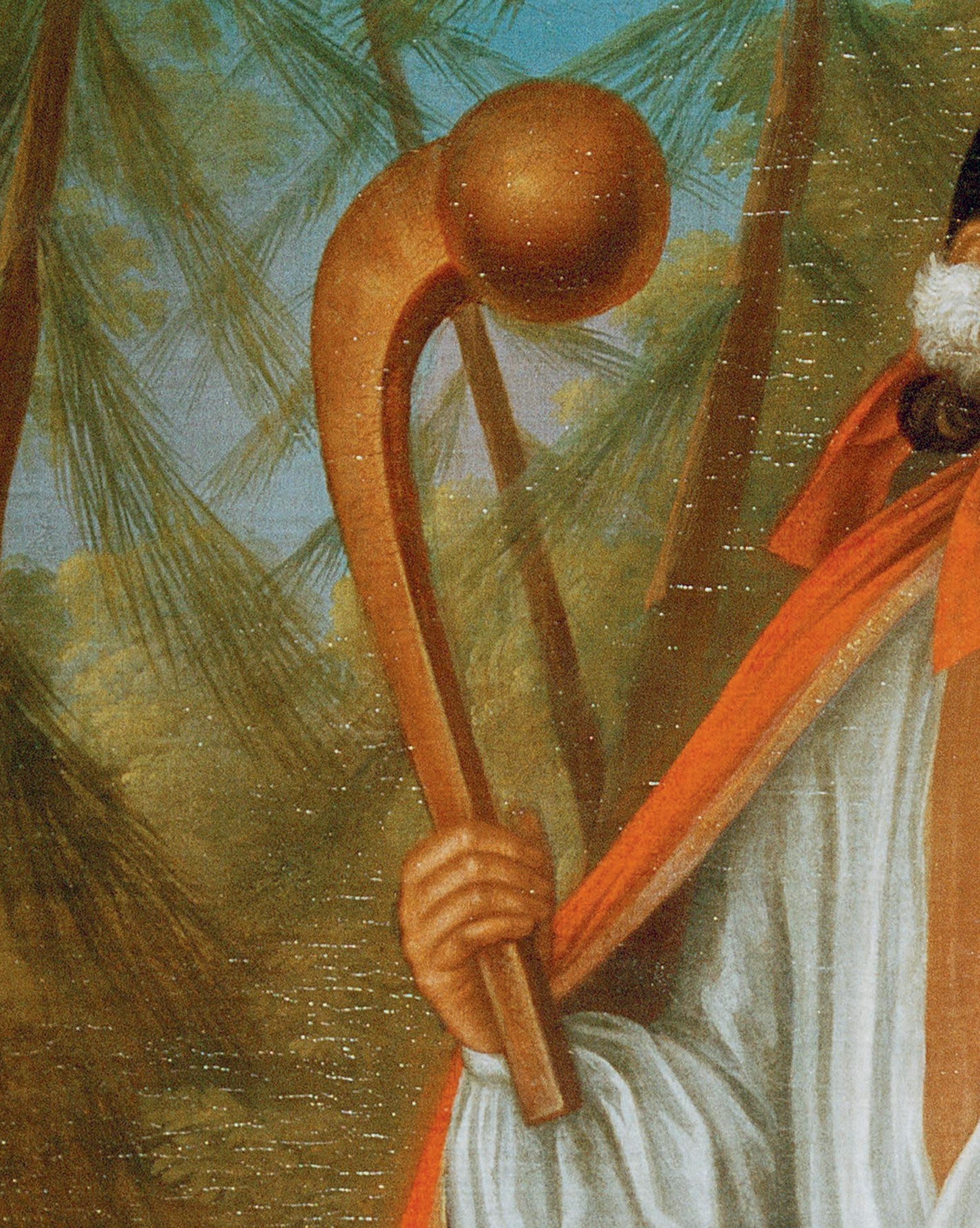


Un collier pour

les lier, un collier

pour les trouver,

un collier pour

les ramener tous

chez eux 
Pendant plus de deux cents ans, les colliers de wampum ont entretenu des relations étroites avec la guerre et les casse-têtes chez les Iroquoiens. Ce texte se propose d'en expliquer la fonction à partir de l'étude de quelques-uns des plus anciens casse-têtes connus à ce jour appartenant à des collections parisiennes. Les wampums étaient nécessaires pour compenser la perte d'un proche. En adoptant leurs prisonniers, les Premières Nations parvinrent à lutter contre la baisse démographique dans leurs rangs. Les colliers de wampum teintés de rouge ont disparu mais leur souvenir est resté, car ils identifiaient publiquement les chefs de guerre. Tombés en désuétude après la Révolution française, les casse-têtes collectionnés sous l'Ancien Régime, extrêmement bien conservés, offrent une occasion unique de se pencher sur le passé. Ils sont le témoin de pratiques de communication non verbale révolues dont la disparition coïncide avec la perte d'autonomie des Premières Nations.

Les wampums et la guerre offrent toute la matière nécessaire à l'écriture d'un roman palpitant. Rien de plus facile done que d'introduire dans notre sous-titre une référence à l'incontournable Seigneur des anneaux, dotant au passage notre article de la même charge dramatique. Comme chez Tolkien, ce n'est qu'une fois l'objet détruit que la paix se met à régner. Mais nous ne sommes pas dans la Comté et personne ne s'appelle Frodon. Non, nous nous trouvons en Amérique du Nord, chez les Premières Nations, entre le XVII et le XVIII ${ }^{\mathrm{e}}$ siècle. À cette époque, les puissances coloniales que sont la France et l'Angleterre se livrent une guerre sans merci. Si nous ignorons le nom de la plupart des porteurs de wampum, certains ont légué leurs faits d'armes à la postérité. Les colliers peints en rouge sont aujourd'hui très rares : tout comme l'Anneau de pouvoir, ils n'ont pas survécu à leur «utilisation». Mais dans la mesure où ils représentaient à la fois un fardeau et un privilège, les chefs de guerre les ont fait reproduire sur leurs armes et leur équipement.

Afin d'illustrer les liens historiques existant entre les wampums et la guerre, nous nous intéresserons à des casse-têtes datant des XVII et XVIII ${ }^{\mathrm{e}}$ siècles. Certaines de ces pièces collectées sous l'Ancien Régiime se trouvent dans des musées parisiens sans aucune note expliquant leur origine, leur âge ou leur signification. Une étude approfondie de ces armes devrait nous permettre de mieux comprendre l'histoire des wampums et de la guerre chez les Premières Nations d'Amérique du Nord. Si ces objets ne sont pas représentatifs de tous les casse-têtes datant de cette époque, ils sont le témoin d'une profondeur historique commune à d'autres vestiges matériels de la culture nordamérindienne. Avant d'aborder le sujet des wampums et de la guerre, commençons par introduire la zone concernée et la terminologie nécessaire pour saisir les explications à venir.

\section{RÉGION D'ÉTUDE}

Graver des motifs figuratifs et géométriques sur les casse-têtes et les pipes tomahawk était une pratique répandue chez les Premières Nations d'Amérique du Nord (Meachum 2005; 2007 ; King 2007 ; Stolle 2012, 2018). La présence de colliers de wampum - des bandes tissées de perles tubulaires en coquillaǵe blanches et violettes - au sein de ce répertoire pictographique est circonscrite à la zone d'utilisation de ces colliers, qui s'étend de la côte atlantique à l'est aux fleuves Missouri et Mississippi à l'ouest, et des Grands Lacs au nord aux Appalaches au sud (carte 1, p.4). Cette région abrite différentes nations, regroupées par les spécialistes en quatre familles linguistiques distinctes. À l'est, les Iroquoiens (avec les Hurons-Wendat et les Haudenosaunee ou Six Nations iroquoises au nord et les Cherokee au sud) [Forster 1996: 100 sq.]; puis les Algonquiens (subdivisés en trois groupes en fonction de leurs stratégies de subsistance, à savoir orientaux - les Abénakis -, centraux - les Sakias et les Mesquakies - et des plaines - les Blackfoot), qui ne font pas partie de notre étude car ils vivent trop au nord-ouest du Missouri (Goddard 1978: 70 sq.; Forster 1996: 98-100); le troisième groupe comprend les Muskogéens au sud (avec les Chicachas et les Chactas) [ibid.: 109 sq.); et le quatrième les Sioux (avec les Catawba à l'est et les Dakota à l'ouest, par exemple) [ibid.: 100 sqq.). Vu l'immensité de la zone, il est très étonnant que la plupart des armes, alors même qu'on ne sait rien de leur provenance, aient été identifiées comme étant soit iroquoises soit huronnes. L'incrustation de perles, visible sur les représentations pictographiques de colliers de wampum gravées sur les manches et les crosses en bois de ces armes, date de l'époque à laquelle les wampums furent le plus larǵement utilisés. C'est-à-dire du début du XvII siècle, avec l'apparition des premiers colliers de perles tissés, 


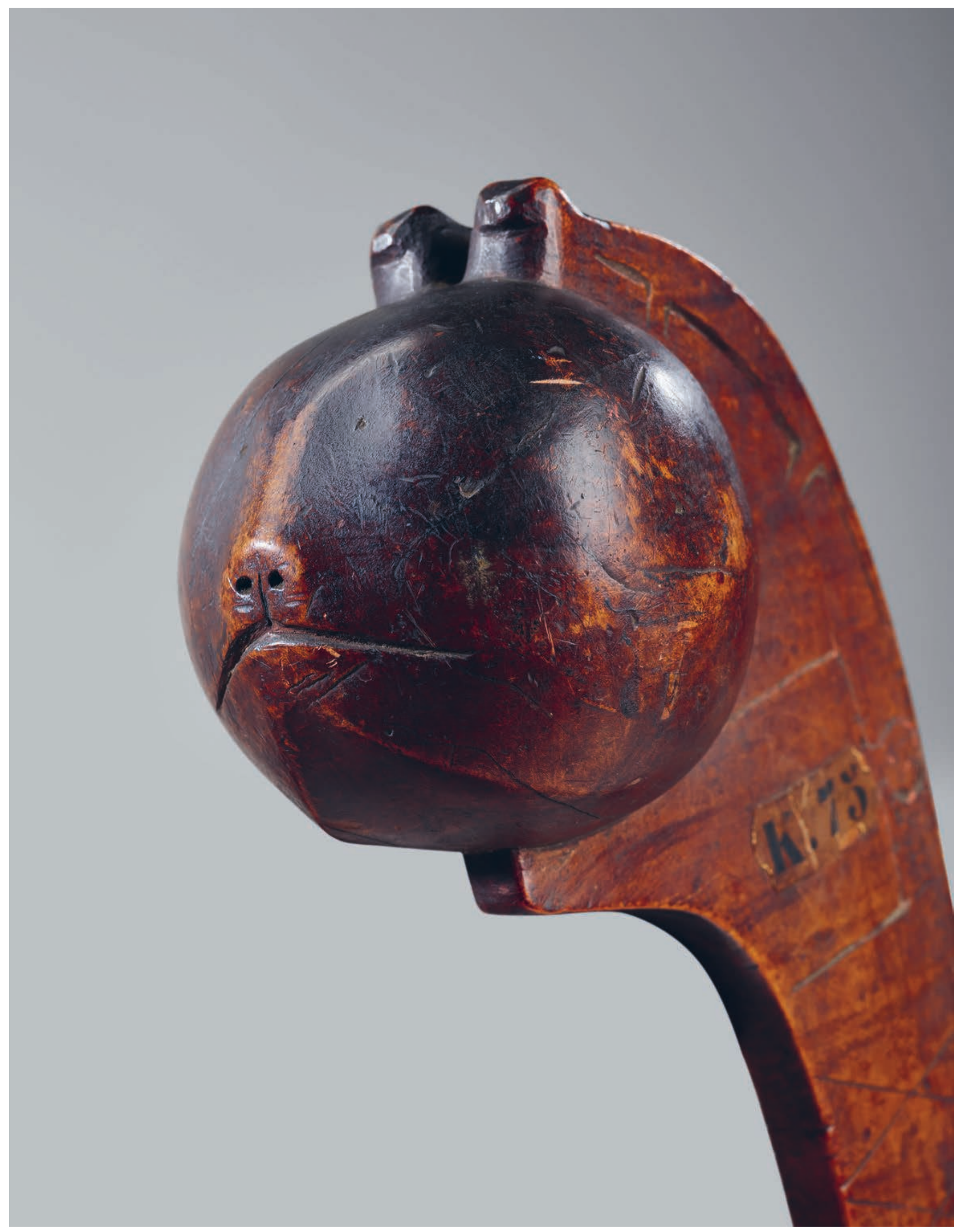

fig. 1. Casse-tête à décor zoomorphe: tête de carcajou (?) et deux loutres. Nord-Est de l'Amérique du Nord, vers 1700 
1. Le second sens en est «le nom d'une grosse pomme sucrée» (Cotgrave 1611: s. p.), qui renvoie à la forme de ces massues.

2. Les Chaouanons, des Algonquiens centraux, se font chasser de leurs terres par les Senecas (des Iroquois de l'ouest) au xvIII siècle. La nation Chaouanon s'installe dans les environs du fort Saint-Louis en environs dufort Saint-Louis du xvII e siècle (Callender 1978: 622 sqq.)

3. À la même époque, le terme «casse-teste» renvoie à des vins qui «donnent des maux de teste» (Furetière 1690: s. p.) Dans de très rares documents, des captifs mentionnent que les massues en question leur ont valu des «maux de teste».

4. La loutre a servi d'animal de clan à des nations
algonquiennes, muskogéennes et sioux. L'absence de ligne («ligne de pouvoir») reliant la tête du propriétaire de l'arme à son protecteur ou manitou nous fait pencher en faveur nous fait pencher en faveur
d'une arme muskogéenne ou sioux - les Algonquiens avaien coutume de recourir à cette ligne. Les Iroquoiens ne se servaient nide cette ligne ni de la loutre commeanima $n$ clan (Meachum 2007: 69; Stolle 2018: 72 sq.).

5. Le X censé représenter des êtres humains sans tête remonte à très loin, à une époque où les têtes des ennemis servaient de trophées (Snow, et al. [dir.] 1996: 88). Ce qu'il y a d'atypique avec cet objet, c'est la présence du même nombre de grands et de petits $X$ sur les deux tranches de la crosse. Habituellement, un seul côté est gravé du nombre de victimes et des symboles renvoyant aux expéditions menées; l'autre est réservé aux raids ou laissé vierge (Meachy (Meachum 2007: 73 sqq.; Stolle 2018: 76 sq.). à leur déclin au début du XIX , période durant laquelle les Premières Nations étaient encore autonomes (voir Lainey; Núñez-Regueiro et Stolle dans ce numéro).

\section{TERMINOLOGIE}

Les documents écrits sont une source précieuse, car ils nous permettent de mieux comprendre l'évolution des langues et de leurs mots, dont nous ne saurious rien sinon. Sous l'Ancien Régime, on utilisait pour désiǵner les armes étrangères des termes qui n'ont plus cours aujourd'hui. Au début de la période coloniale, toutes les armes de combat rapproché répondaient ainsi au nom de «massues ${ }^{1}{ }^{»}, c l u b$ en anglais (Cotgrave 1611: s. p.). À la fin du XvII siècle, la définition s'affine: il s'agit désormais d'une «[a]rme faite d'une grosse pièce de bois, lourde $\&$ grosse par un bout, \& armée de plusieurs pointes. L'arme d'Hercule étoit sa massue» (Furetière 1690: 576). Cette précision nous donne une meilleure idée de la forme de ces armes de combat dont l'extrémité fortement bombée pouvait comporter des saillies. Le terme «massue» conserve son sens pendant tout le XVIII ${ }^{\mathrm{e}}$ siècle (Boyer 1729: s. p.). À la fin du XVII siècle, un nouveau terme apparaît en français : Henri Joutel, explorateur et soldat, recourt au mot «casse-têtes» lorsqu'en mars 1687 il apprend que les Chaouanons ${ }^{2}$ coupent du bois pour fabriquer ces «espèces de massues que les Sauvages font et dont ils se servent dans leurs surprises pour casser la teste ${ }^{3}$ » (Marǵry 1876: 328). Le mot «casse-tête», comme son nom l'indique, renvoie donc à un type précis de massue. Mais à quoi ces armes ressemblaient-elles exactement? Deux descriptions nous aident à nous en faire une idée. La première date d'avant 1701 et parle de «bâtons qui ont la figure de coûtlas (Bacqueville de La Potherie 1722, t. III : 96); la seconde, qui date de 1709 , indique qu'il «a la figure d'une machoire, est fait d'un bois dur et pesant, et a une masse ou une boulle au bout» (Rochemonteix [éd.] 1904: 76). Il semblerait donc que le terme «casse-tête» ait à l'époque renvoyé à deux types d'armes de choc en bois, l'une plus volumineuse en son extrémité et sculptée en forme de boule au niveau du point d'impact, l'autre en forme de croissant de lune, sur le modèle du coutelas ou du sabre court européen. Les Canadiens francophones utilisent aujourd'hui ce terme dans ses deux acceptions pour parler des massues de guerre des Premières Nations. Dès 1744 , le mot «casse-tête» gagne un nouveau sens : Dumont de Montigny, un officier français envoyé en Louisiane, l'utilise pour décrire «une petite hache portative» (Bnf Ms 3459, Dumont de Montigny 1744 : fol. 169). Il évolue à nouveau dans la seconde moitié du $\mathrm{XVIII}^{\mathrm{e}}$ siècle, après que les pipes tomahawk sont devenues une marchandise en Amérique du Nord. Pierre Marie François Vicomte de Pagès nous en fournit une excellente description en 1767 à l'occasion de son séjour en Louisiane: "[1]e casse-tête [du chef] est une espèce de hache d'armes, dont le manche creux communique au dos de la hache, sur lequel est attachée une tête de pipe en fer» (Bérenger 1790: 12).
L'évolution de ce terme, qui renvoie successivement à une massue, à une petite hache et à une pipe tomahawk, rend bien compte de la difficulté d'interpréter des documents historiques écrits lorsque aucune description additionnelle n'est fournie. Parallèlement, elle nous en dit long sur la transformation des armes offensives au sein des Premières Nations. Les nouveaux outils multifonctionnels, tels que la hache, à la fois outil et arme, ou encore la pipe tomahawk - qui revêt même une troisième fonction, celle de pipe à tabac - finissent par remplacer les anciennes armes en bois qui n'étaient rien d'autre que des armes.

\section{LES LIENS ENTRE LE PLUS ANCIEN CASSE-TÊTE ZOOMORPHE À TÊTE SPHÉRIQUE ET UN COLLIER DE WAMPUM}

Parmi plus de cent vingt casse-têtes datant des $\mathrm{XVII}^{\mathrm{e}}$ et $\mathrm{XVIII}^{\mathrm{e}}$ siècles, il n'en est qu'un à notre connaissance dont la tête arrondie ait des traits zoomorphes (fig. 1). Il est fabriqué dans une seule pièce d'érable, le nœud de souche dans lequel est taillée la boule figurant les traits d'un prédateur. La tête ronde, les yeux profondément enfoncés dans les orbites et la séparation entre le museau et la lèvre supérieure ne laissent guère de doute: le prédateur en question est un carcajou ou un pékan. Au-dessus de la boule, la crosse se prolonge et se divise en deux branches sur lesquelles est gravé un couple de mammifères. Les corps fins et lonǵs, de petite taille, dotés d'une queue et de courtes pattes évoquent des loutres. Les deux tiers de la crosse sont rectangulaires et le dernier tiers consiste en un manche ovale destiné à être saisi à deux mains. Des motifs géométriques sont entaillés sur les tranches externes et des éléments figuratifs gravés plus ou moins finement ornent le côté gauche. Ainsi, on peut observer les contours rectangulaires d'un torse humain surmonté d'une tête ronde décorée de trois points peints en rouge représentant les yeux et la bouche; il est probable qu'il s'agisse du propriétaire de l'arme. Les deux prédateurs se faisant face incisés sur sa poitrine (Meachum 2007 : 69-70; Stolle 2018: 72-73) ressemblent aux loutres gravées sur les branches de la crosse, à ceci près que leur corps est plus trapu et que leurs oreilles sont plantées sur le dessus du crâne. Leur queue longue et fine rappelle davantage celle des carcajous que celle des pékans, plus courte et touffue. Ils sont les protecteurs du propriétaire de l'arme qui se les est tatoués sur la poitrine et les a fait graver sur la boule de son casse-tête, la partie directement en contact avec l'ennemi. Les loutres renvoient pour leur part à sa famille ou à son clan ${ }^{4}$. Au-dessus de cette silhouette humanoïde, treize X très légèrement incisés dans le bois sont à peine visibles. Le même nombre de $\mathrm{X}$ est gravé plus profondément sur la tranche gauche de l'arme, s'étendant de la gauche de la silhouette jusqu'au milieu de la poignée. Chaque X représente un être humain sans bras ni tête. Le nombre de X renvoie au nombre d'ennemis tués ou scalpés par le propriétaire de l'arme, et tous sont reliés par une ligne ${ }^{5}$. De légères marques sont visibles à côté du dernier $\mathrm{X}$, une suite de lignnes parallèles dont la plus basse est surmontée 
d'un crochet. Ce motif est particulièrement intéressant car il représente un collier de wampum. Comment les guerriers liaient-ils leurs armes aux wampums?

\section{COLLIERS DE WAMPUM ET GUERRE}

La première représentation connue d'un wampum de guerre date de 1666 environ. Il s'agiit d'une copie française d'un dessin amérindien (fiǵ. 2). Le texte qui l'accompaǵne commence comme suit: «[1]a Nation Iroquois est composé » (Anom C 11, A 4 : fol. 264 ; voir O'Callaǵhan 1849: $3^{6}$ ). Grâce aux neuf clans représentés de profil en haut du dessin (Fenton 1978: 299), on lui attribue une origine seneca - les Senecas sont une des nations iroquoises (Haudenosaunee) vivant le plus à l'ouest. Les animaux de chaque clan 7 portent une arme de combat rapproché - casse-têtes, petites haches et coutelas. En bas de la reproduction, au centre, deux animaux se font face; la lettre $\mathrm{G}$ renvoie au commentaire suivant "Conseille de Guerre entre La famille [clan] de L[']ours et celle du Castor ils sont frere» (Anom C 11, A 4 : fol. 266r; voir O'Callaghhan 1849: 7). À gauche, un ours (H) offre à un castor (I) un collier de wampum, identifiable grâce aux franges visibles à chacune de ses extrémités. Le collier (L) est décrit comme suit: "un collier qu[']il tient dans ses pattent [pattes] pour aller venger la mort de quelqu'un et il est apres conserver de cela avec son frere le castor» (Anom C 11, A 4 : fol. 266r; voir O'Callaghan 1849: 7).

Il incombait au proche d'une personne décédée de présenter un collier de wampum à un membre d'un autre clan pour compenser la mort de la personne en question, comme le montrent l'ours et le castor dans cet exemple d'un conseil de guerre. Cette brève description en dit long sur les Iroquois d'antan (les actuels Haudenosaunee). Tout d'abord, il fallait compenser chaque décès, qu'il soit de cause naturelle ou violente; ensuite, le remplaçant devait être quelqu'un d'extérieur au clan qui ne soit pas touché par le décès et ne soit pas en période de deuil; enfin, il fallait avant toute autre action présenter un collier de wampum. Afin d'illustrer le besoin de condoléances que chaque décès provoquait, citons ici les mots particulièrement éclairants de Joseph François Lafitau ${ }^{\mathbf{8}}$, un jésuite ayant vécu parmi les Mohawks de Sault-Saint-Louis (actuellement Kahnawake) entre 1711 et 1717 :

Les familles, [clans...], ne se soutiennent que par le nombre de ceux qui les composent, soit homes, soit femmes; c'est dans ce nombre que consistent leurs forces \& leurs principales richesses. La perte d'une seule personne est une grande perte, mais une perte qu'il faut nécessairement réparer, en remplaçant cette personne qui manque, par une ou par plusieurs autres, selon que la personne qu'on doit remplacer, étoit plus ou moins considerable»

(Lafitau 1724, t. II : 163)
Voilà pourquoi on incita les Iroquoiens, les Algónquiens, les Sioux et les Muskogéens à se faire la guerre: parce qu'ils n'étaient guère nombreux et que les multiples batailles entre les différentes puissances coloniales européennes de l'époque les avaient largement affaiblis ${ }^{9}$. Si elles n'avaient pas décidé d'adopter des captifs autochtones ou euro-américains, nul doute que la plupart des nations auraient disparu. À chaque fois qu'un individu mourait ou, pour reprendre l'expression métaphorique d'alors, se «couchait sur sa natte ${ }^{10}$ », la mère du clan s'en remettait à l'homme le plus capable, souvent un grand guerrier, et lui présentait un collier de wampum pour qu'il lève les armes et compense cette perte (Havard 2007 : 109 sqq.).

\section{LEVER LES ARMES ET SE SERVIR D'UN COLLIER DE GUERRE}

Comme nous l'avons observé ci-dessus avec la reproduction d'un dessin iroquois datant de 1666, on présentait un collier de wampum à quelqu'un pour qu'il lève les armes. Les femmes ne pouvaient ni déclarer la guerre ni conclure des traités de paix, ce qui veut dire qu'elles n'étaient pas autonomes (Lafitau 1724, t. II : 164). Mais elles n'en jouaient pas moins un rôle en coulisse et pouvaient être à l'origíne d'une expédition en présentant un collier de wampum à un guerrier (voir ci-dessus) ${ }^{11}$. Le futur chef organisait ensuite les cérémonies requises, que nous ne détaillerons ni n'analyserons ici de peur de nous éloigner du sujet. Citons tout de même Lafitau qui, lorsqu'il parle des guerriers iroquoiens et de leurs obligations, fournit une courte description de la procédure à suivre:

\section{Celui qui a envïe de lever un parti, ou qui est ainsi engage à le faire, fournit un collier, ou bien s'il la reçû, il le montre à ceux qu'il veut enroller dans son expedition, comme le signal de son engagement, \& du leur (Lafitau 1724, t. I: 167; Fenton 1998: 26 sq. ${ }^{12}$ ).}

Par conséquent, un collier de wampum pouvait être présenté dans deux cas de figure: par une femme ou un membre du clan comme invitation à compenser la mort d'un proche ou par le futur chef d'une expédition.

Commençons par le dernier acte que réalisait le chef en question avec son collier de wampum afin de rallier des guerriers pour son expédition. Pierre Pouchot ${ }^{13}$, le commandant du fort Niagara, entretenait des relations cordiales avec les autochtones; en effet, ils étaient des alliés de choix pour la France dans sa guerre contre la Grande-Bretagne:

\footnotetext{
Le chef de guerre [...] annonce son projet, de l'air le plu menaçant qu'il peut, \& invite ceux qui ont du courage à le suivre. Il finit par jeter un collier de porcelaine noire, barbouillé de rouge, avec dedain à terre, invitant ceux qui ont du cœur à le relever, \& annonçant qu'il le destine pour celui qui se montrera le plus courageux. (Pouchot 1781: 339 sq. ${ }^{\mathbf{1 4}}$ )
}

6. Edmund Bauley O'Callaghan a été le premier à en publier une traduction en anglais qui peut différer de l'original (1849. 3 sqq.; 1850: 11 sqq.). William N. Fenton et William C. Sturtevant ont attribué cette copie à Pierre-Joseph-Marie Chaumonot, un jésuite ayant résidé au Canada entre 1639 et1693, l'année de sa mort. Il était missionnaire auprès des Hurons, des Neutres et des Cinq Nations iroquoises aux environs du lac Ontario (Drolet 1989; Fenton 1998: 25 sq.).

7. À l'exception de la «famille de la pomme de terre», à la sixième place, qui ne porte aucune arme (Anom C 11, A 4: fol. 263). Ce clan fut identifié en 1736 par un autre Français, vraisemblablement LouisThomas Chabert de Joncaire, qui épousa une femme seneca après un épisode de captivité (O'Callaghan 1849: 21). La pomme de terre en question est l'Apios americana ou haricot pomme de terre (Girard 2020). Ce clan, disparu au xvIII' siècle, ne fait aujourd'hui plus partie de la nation Seneca.

8. Les témoignages de Lafitau sont malheureusement truffés d'observations réalisées par Julien Garnier, son mentor, lui-même missionnaire auprès des Onneiouts, Onontagués et Senecas dans les années 1670 , qui s'inspira beaucoup de textes publiés sur les Hurons-Wendat. Seules les descriptions d'une réunion de condoléances, de la construction d'un canoë et d'une maison longue sont basées sur ses propres observations des Mohawks de Sault-Saint-Louis, actuellement Kahnawake (Lafitau 1974 [1724]: XXIX sqq.). Ce témoignage demeure précieux étant donné le respect infini qu'il voue à la culture amérindienne: il ne tarit pas d'éloges sur la maîtrise de soi du peuple qu'il côtoie, sa politesse, son hospitalité et son mode de vie pacifique. Si ses écrits prêtent aujourd'hui à polémique, une étude plus pointue et contextualisée à la fois de ceux qui furent publiés et des autres reste à faire.

9. Si nous prenons l'exemple des Hurons de Lorette, qui sont aujourd'hui les Hurons-Wendat de Wendake, nous savons qu'ils passèrent de soixante guerriers en 1736 (O'Callaghan 1849: 17) à quarante en 1752 (Franquet 1889: 107), soit deux ans avant le début de la guerre de la Conquête (1754-1760)

10. Les nattes, faites de fibres végétales, servaient pour s'asseoir ou dormir. Chaque guerrier avait la sienne et l'emportait partout où il allait. À sa mort, il était enterré avec (Lafitau 1724, t. II: 195: 1974-1977 [1724], t. II: 115). Cette pratique est de mise dans toutes les familles linguistiques mentionnées.

(Voir suite des notes p.68) 


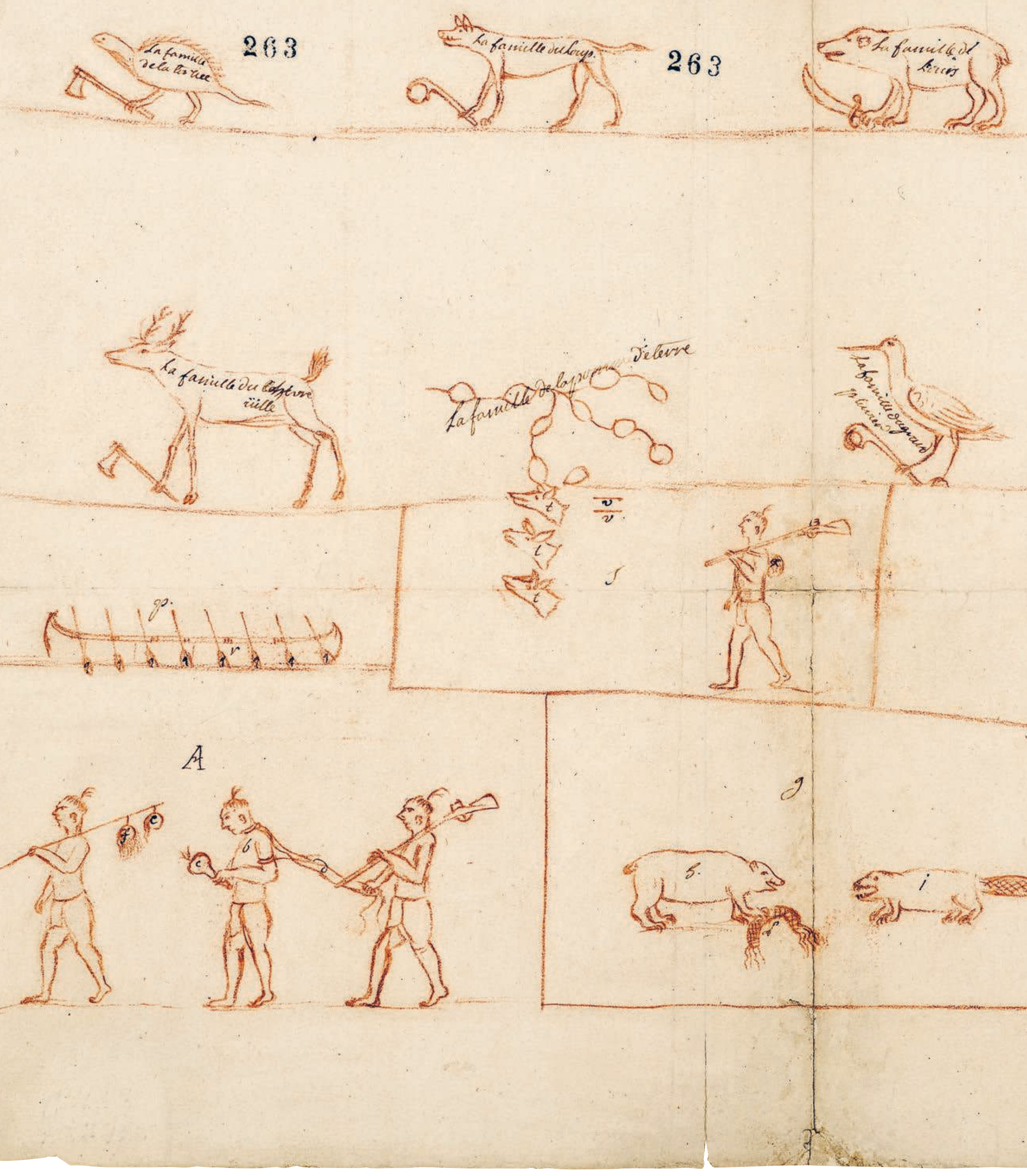




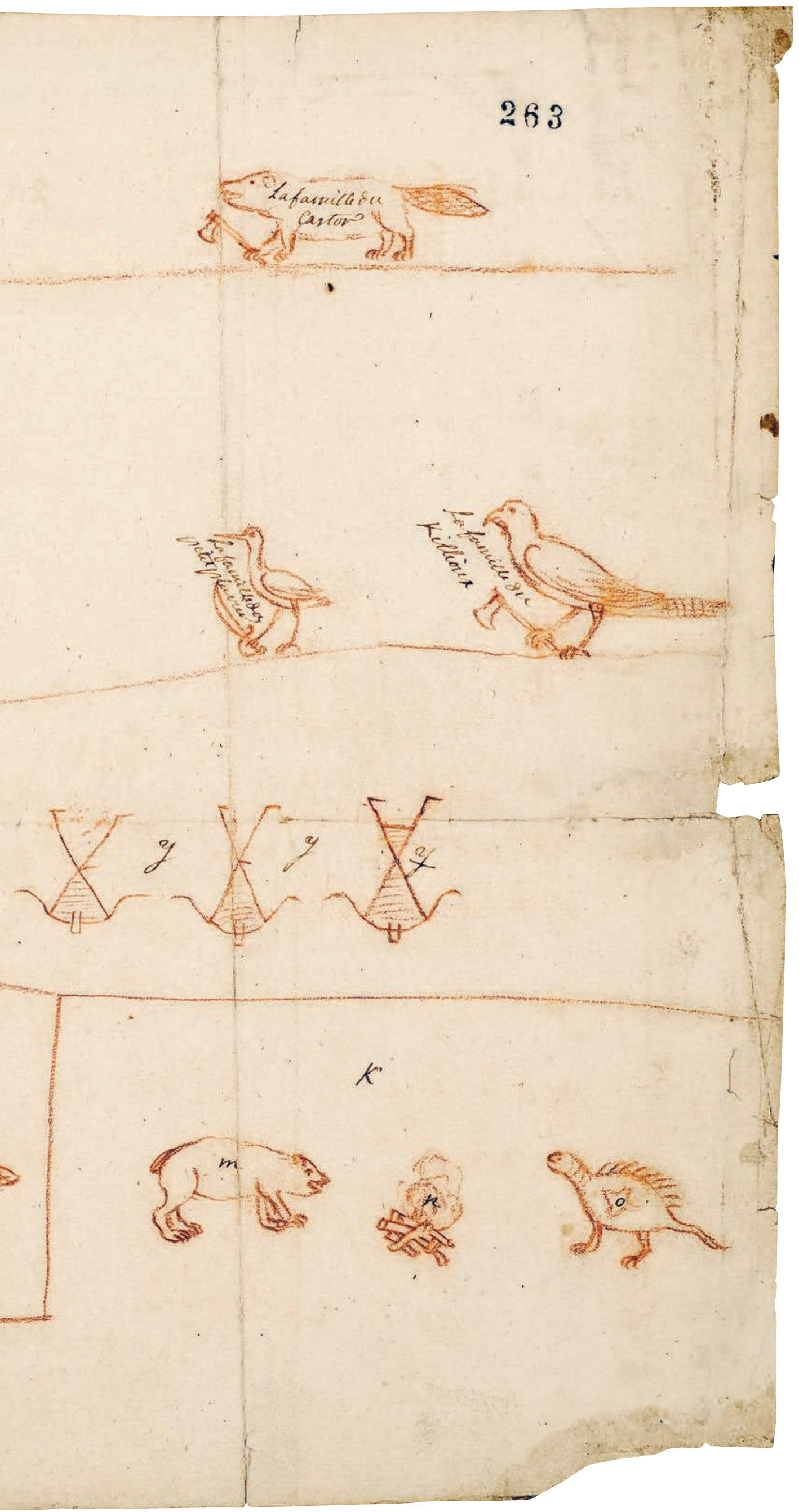

fig. 2. Pierre-Joseph-Marie Chaumonot (attribué à) Reproductions de pictogrammes autochtones indiquant notamment des noms de clans attribués à la Nation Seneca, vers 1666. Dessin à l'encre sur papier, $49 \times 35 \mathrm{~cm}$ Archives nationales d'Outre-Mer, Aix-en-Provence, cote ANOM COL C11 A $2 \mathrm{~F}^{\circ} 263$.

Ces illustrations accompagnent la section du manuscrit «La Nation Iroquoise» où Chaumonot décrit l'organisation sociale en clans des Seneca et les faits guerriers établissant le statut et le rang. 


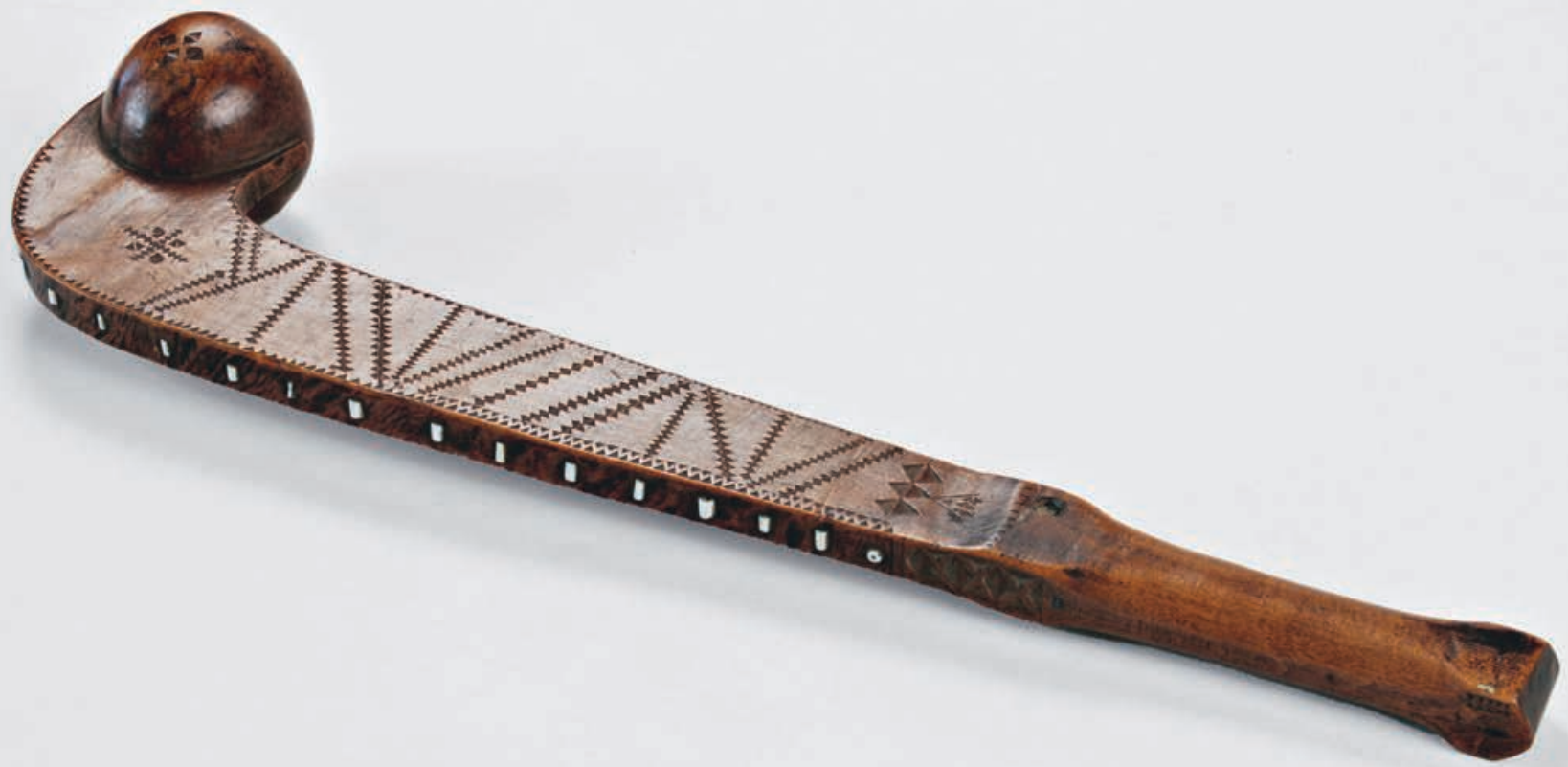

fig. 3. Casse-tête incrusté de wampum blanc et violet. Nord-Est de l'Amérique du Nord, avant 1688. Érable, escargot de mer, palourde (Mercenaria mercenaria), traces de pigment noir,

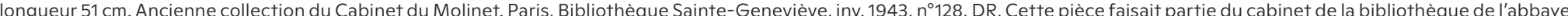

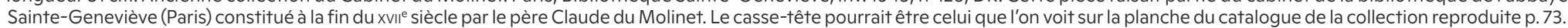

11. Sir William Johnson, surintendant des affaires indiennes dans les districts britanniques d'Amérique du Nord, avait conscience que jouaient les femmes au sein des Six Nations iroquoises. Il épousa Molly Brant, la sœur ainée de Joseph Brant, qui était née dans une famille de mères de clan où ce titre se transmettait de mère en fille. Voilà ce que le lieutenant-colonel Tench Tilghman, secrétaire et aide de camp de George Washington, écrit sur lui en 1775 , soit un an après sa mort, au cours d'une de ses visites aux Mohawk Canajohare: Canajohare: «[i]l savait que les femmes régnaient sur la politique des sauvages, tout comme cela se fait dans les régions du monde les plus raffinées.» Tilghman avait conscience que uson influence bien des ennuis car nous savons qu'elle travaille d'arrache-pied à ce que le congrès d'Albany n'ait pas lieu, toute dévouée qu'elle est à la cause de Guy Johnson», le neveu de William, qui le remplaça à son poste auprès de la Couronne britannique (Harrison [éd.] 1876: 83, 87)

12. En 1639 , soit plus d'un siècle plus tôt, le jésuite Paul le Jeune décrit quelque chose de similaire chez les Hurons: «Quãd quelque
Le collier de wampum noir ou violet était vraisemblablement peint en rouge ou vermillon, comme le mentionnent certains documents (NYCD 1856, t. VII : 385). La couleur signifiait que l'objet passait du statut de parole sincère à celui de collier de guerre. Dans l'histoire iroquoienne, le rouge symbolise en effet les «états anti-sociaux», elle est la couleur du sanǵ et de la vie (Hamell 1992: 456 sq.) ${ }^{15}$.

C'est ainsi que commençaient les guerres intertribales et confédérales. Louis Antoine de Bougainville, l'aide de camp du marquis de Montcalm, lui-même commandant des troupes du Canada lors de la guerre de Conquête en Amérique du Nord (1754-1760), écrit en 1757 :

Le terme de délier veut dire renvoyer et est pris de ce qu'on invite les Sauvages à une expédition en leur présentant un collier, et lorsqu'ils l'acceptent c'est un gage qu'ils se lient pour suivre le général au nom duquel il est présenté et pour l'expédition qui leur est annoncée.

(Hamilton [éd.] 1964: 98; Bougainville 2003 [1756-1760] : 176)

Voilà qui nous rappelle les expéditions auxquelles différents clans participaient ainsi que la fonction des colliers de wampum : lier deux partis, s'assurer qu'ils tiendront leur engagement l'un envers l'autre. S'ils partagent la même fonction, les colliers de guerre étaient bien plus grands que ceux échangés entre clans. La même année, Bougainville note que «le marquis de Vaudreuil [gouverneur de la Nouvelle-France] a fait mettre aux pieds des Onnéyotes ${ }^{16}$ un collier de guerre de 6000 ǵrains, mataché de rouge et sur lequel est représentée une hache» (Hamilton [éd.] 1964: 111; Bougainville 2003 [1756-1760] : 186). On offre des colliers de plus grande taille et donc de plus grande valeur pour attirer l'attention d'une nation et l'inviter tout entière. Contrairement aux colliers échangés pour des expéditions de moindre ampleur, les colliers de guerre étaient souvent décorés d'une hache blanche. À l'époque coloniale, on appelait ces colliers peints en vermillon des «hachettes». Le collier de Vaudreuil est une nouvelle fois mentionné en 1759, juste avant un affrontement, lorsque le chef Conoquieson, un Onnéyote, le tend à sir William Johnson en prononçant le discours suivant: «Je vous présente une hachette (un collier de wampum avec un motif de hache) pour que vous vous en serviez contre les Anǵlais. » (NYCD 1856, t. VII : 385 17) Ces colliers de guerre étaient remis à la nation la plus puissante de la coalition pour la simple et bonne raison que c'était celle qui fournissait le plus grand nombre de guerriers ${ }^{18}$. Ce geste nous rappelle le passage cité 


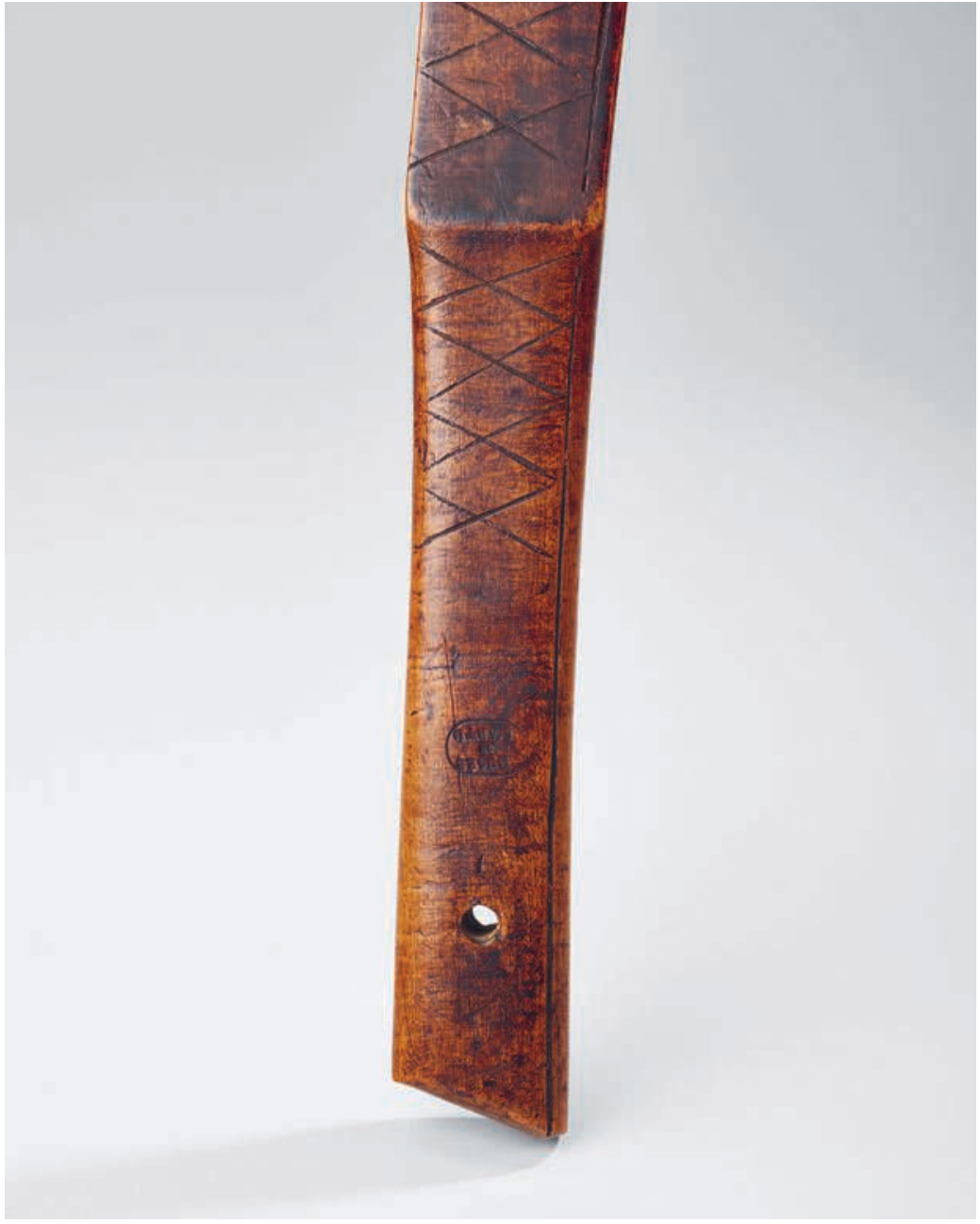

fig. 4. Casse-tête, Nord-Est de l'Amérique du Nord, vers 1700. Érable, traces de pigments rouges et noires, étiquettes en papier, étiquette estampée. Longueur $59 \mathrm{~cm}$, inv . 71.1917.3.11 D. Ancienne collection du Prince de Condé, château de Chantilly ๑) musée du quai Branly-Jacques Chirac, photo Pauline Guyon.

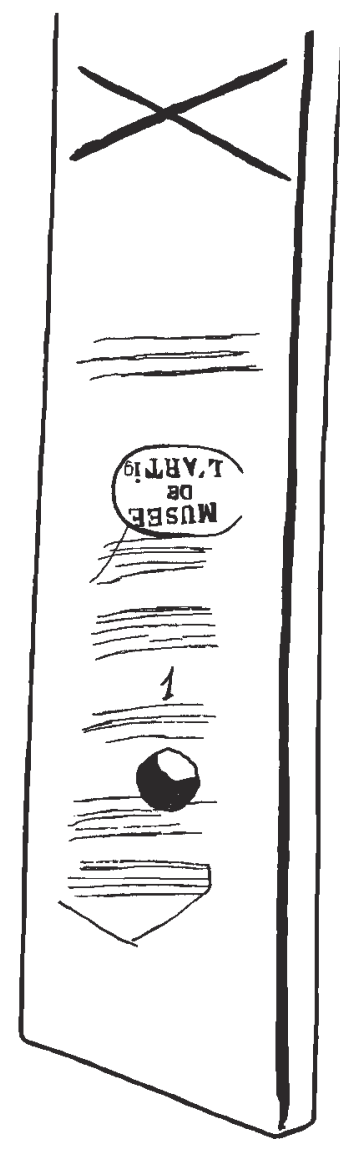

fig. 5. Relevé du décor incisé visible sur le manche du casse-tête 711917.311 D : figuration d'un collier de wampum et de nattes servant de tapis (c) Nikolaus Stolle. ci-dessus expliquant que des colliers étaient présentés aux guerriers les plus courageux lors de conflits intertribaux. Dans les deux cas, ils récompensaient donc la force et le courage. Si quelqu'un ramassait un collier jeté à terre, cela signifiait que lui ou sa nation acceptait de participer à l'expédition. Ces obligations liées aux colliers nous éclairent sur les raisons qui ont pu pousser les Amérindiens à vouloir se débarrasser des colliers de guerre tant ils étaient synonymes de souffrances et de mort. Citons les mots de Tarhe, chef des Wyandots [Hurons-Wendat], en 1795:

\section{Brûlez-le ou transformez-le en collier pour l'offrir à une jolie squaw. En modifiant son apparence et ses motifs originels, vous pourrez être sûrs qu'on ne le reconnaîtra jamais. Il nous a causé tellement de mal- heurs que je suis heureux de m'en séparer. (Clark et Lowrie [éd.] 1832 [1789-1815] : 574)}

\section{MORT OU VIF}

Le plus grand honneur pour un guerrier était de capturer un prisonnier. En effet, c'était une tâche extrêmement ardue que de réussir à ramener un captif vivant sans se faire remarquer par le camp adverse.
Un prisonnier adulte cherche normalement à s'échapper, et les Euro-Américains n'étaient guère résistants face aux épreuves imposées par une marche forcée - plusieurs jours sans manger, presque sans boire ni dormir, afin de ne pas être repéré par l'ennemi (Snow et al. [dir.] 1996: 64-66). Les scalps ${ }^{19}$, qu'ils proviennent d'individus vivants ou morts, offraient un piètre substitut de prisonnier, l'objectif principal des guerriers étant de renforcer leur communauté et de remplacer les morts par des captifs. Dès qu'un guerrier rejoignait son territoire ancestral, il s'arrêtait pour indigéniser son captif, lui couper les cheveux, le vêtir à la mode de sa tribu. À cette occasion, on attachait autour du cou du prisonnier des colliers de wampum peints en rouge. Pouchot précise qu'ils se portaient «comme nos Dames le portent» (Pouchot 1781: 351; Snow et al. [dir.] 1996: 65), ce qui veut dire qu'ils étaient de petite taille 20 .

Heureusement pour nous, le commandant est l'un des rares à avancer une explication à ce geste: le collier est «ce qui marque son esclavage» (ibid.). Cette interprétation d'une pratique iroquoienne et algonquienne ${ }^{21}$ rend possible la comparaison avec d'autres rites où un wampum était également attaché autour du cou 22. Chez les Haudenosaunee (Six Nations iroquoises), on dit encore d'un individu qu'il portera «autour de son cou» le nom que le clan lui donnera brave homme a esté massacré par leurs ennemis, s'il auoit quelque Collier de porcelaine ou autre chose de valeur, ses [154] amis l'offrentà quelque bon guerrier, ou luy font quelque present de leurs propres moyens, si cét homme les accepte avec le nom du defunct qu'on luy donne publiquement, il s'oblige d'aller à la guerre.») (JR 1898, t. XVI: 203)

(Voir suite des notes p.71) 


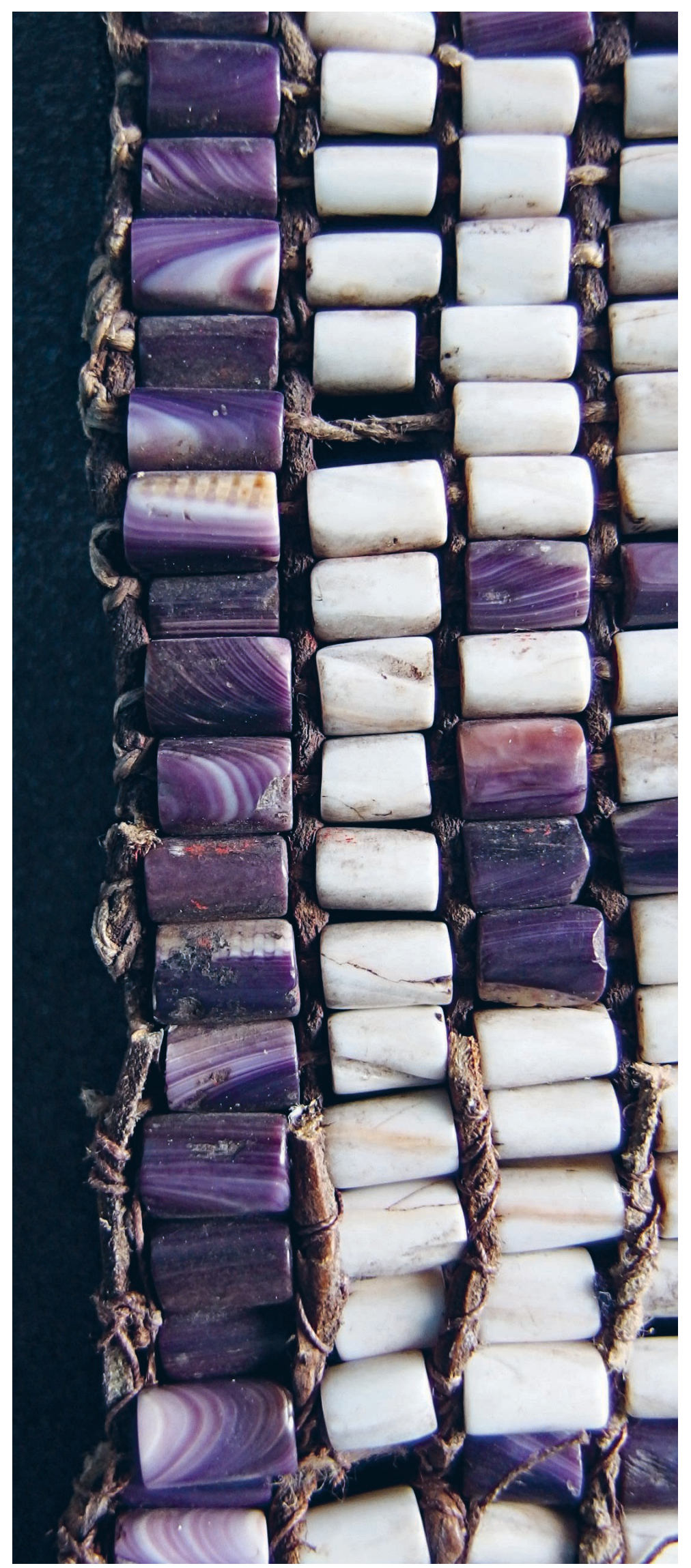

fig. 6. Détail d'un collier de wampum montrant des traces de pigment rouge sur l'une des perles

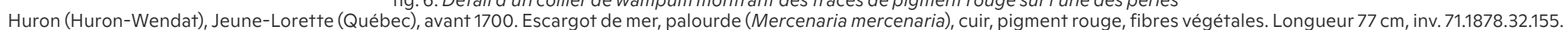
Ancienne collection du Cabinet des médailles, BnF @ musée du quai Branly-Jacques Chirac. 
(Shimony 1994: 214) ${ }^{23}$ : de cette manière, il intègre la nation de la communauté, même si, en sa qualité de captif, il n'en est qu'un membre subordonné. Mais ce n'est pas parce que la décision d'adopter un prisonnier a été prise et qu'un wampum a été attaché autour de son cou que la situation est irrévocable. Il arrive que la famille censée accueillir ce nouveau membre le rejette. Le captif est alors remis à de jeunes guerriers et à d'autres membres de la nation pour être torturé. En cas d'échec d'une expédition, un prisonnier devait être sacrifié, surtout si le chef ou des individus de haut ranǵ avaient perdu la vie dans l'affrontement (Pouchot 1781: 357). En cas d'adoption, le wampum était détaché et offert par le chef de l'expédition au guerrier le plus courageux, un acte symbolique pour encourager les guerriers à se comporter en héros. À partir du moment où le collier n'avait plus d'utilité, son nouveau propriétaire pouvait le détruire, c'est-àdire utiliser ses perles autrement, comme dans l'exemple précédemment cité du chef Tarhe des Wyandot (les Hurons-Wendat actuels), où il incite à le transformer «en collier pour l'offrir à une jolie squaw» (Clark et Lowrie [éd.] 1832 [1789-1815] : 574). C'est la raison pour laquelle des perles tachées de rouge figurent sur des colliers notamment reliǵieux ou votifs (fig. 6).

\section{SYMBOLE DU STATUT}

Louis-Henri de Bauǵy, aide de camp du marquis de Denonville, écrit le 7 juillet 1687 en pleine campaǵne contre les Iroquois que «nos sauvages ${ }^{24}$ ayant mis a terre ont découvert les pistes de 5 Hiroquois, le casse teste d'un a esté trouvé qui a esté reconnue pour estre d'un Onontague [Onondaga]» (Baugy 1883: 92). C'est une précision intéressante car elle nous apprend qu'à l'époque, il était possible d'identifier à quelle tribu appartenait une arme de combat, un savoir perdu depuis. Ces armes étaient donc des symboles lisibles grâce non seulement à leur forme mais aussi aux motifs qui les ornaient. Si des sources écrites précisent que les guerriers gravaient et peignaient leurs exploits sur des troncs d'arbre débarrassés de leur écorce en des endroits choisis qui servaient ainsi de points de repère, il semblerait que leur support préféré ait été leurs armes. Ils se faisaient également tatouer ces scènes, agrémentées de leur esprit protecteur et des symboles de leur clan, à des endroits visibles sur le corps (Meachum 2007; Stolle 2018: 70). C'est ce que Radisson raconte sur son père, un chef de guerre de premier plan qui «tua 19 hommes de ses propres mains, ce pour quoi il reçut sur sa cuisse droite autant de marques qu'il en avait tué» (Snow et al. [dir.] : 81).

Certains des plus anciens casse-têtes autochtones d'Amérique du Nord sont conservés en France. Ils sont la preuve de nombreuses batailles et alliances ainsi que le témoin des exploits de leurs propriétaires. La plupart des entailles gravées sur ces casse-têtes datant des XVII ${ }^{\mathrm{e}}$ et XVIII ${ }^{\mathrm{e}}$ siècles ne sont pas très profondes, ce qui explique pourquoi il est aisé de passer à côté ${ }^{25}$. La fiǵ. 5 donne à voir les incisions pratiquées sur le casse-tête décrit ci-dessus (fig. 4). Les ǵuerriers des Premières Nations documentaient donc leurs actions soit en les dessinant, soit en les gravant côte à côte en superposition. Par chance, la copie française du dessin seneca mentionnée plus haut fournit une explication que Lafitau et d'autres ont validée plus d'un siècle et demi plus tard (Meachum 2007 : 70 sq.). Le rectanǵle, parfois rayé de barres comme dans le cas présent, représente une natte, à laquelle Lafitau attribue la fonction suivante: «[e]n effet la natte est encore aujourd'huy le symbole qu'ils representent dans leurs peintures Hieroǵlyphiques pour désigner le nombre de leurs campagnes. » (Lafitau 1724, t. II : 195) ${ }^{26}$. À en croire cette explication, le propriétaire de ce casse-tête aurait donc participé à six campaǵnes et même dirigé la dernière, pour laquelle on lui remit un collier de wampum - selon les mots de Lafitau, «il commandé le parti» $(1724,3$ : 39, pl. 3). Le wampum consacre son rôle de chef, d'individu occupant une position honorifique au sein de sa communauté et méritant done de peindre, de graver ou de tatouer ses hauts faits sur son corps et ses possessions.

L'histoire de ce spécimen remarquable nous renvoie à la première moitié du XviII ${ }^{\mathrm{e}}$ siècle. L'objet provient des collections de Louis V Joseph de Bourbon, prince de Condé (1736-1818), qui hérita d'une des plus riches collections d'objets d'histoire naturelle, dont quarante-neuf pièces ethnographiques décrites en 1793 comme «quelques parties d'armures de Sauvage comme un carquois plein \& fleches, et une masse» (AN AJ/15/836 Collections de Chantilly: s. p. ; voir: Bandau et al. [dir.] 2010: 56; Lacour 2019: 122 sq., 164 et 212). La collection du prince de Condé a été confisquée puis intégralement transférée au Muséum national d'histoire naturelle où elle occupe deux pièces; en 1798, les armes et les armures rejoignent quant à elles le dépôt de l'artillerie (AN F/17/3978, s. p.). Le casse-tête qui nous intéresse et un autre figurent dans la liste des objets transférés avec la mention suivante: «deux casse-têtes de Sauvages». La description donnée dans la deuxième édition du guide du musée de l'Artillerie paru en 1826 est la suivante: «No. 2 Deux casse-têtes ordinaires des sauvages du Canada» (Anonyme 1826: 38 27).

Non contents de graver et de peindre des colliers de wampum sur leurs casse-têtes, les guerriers incrustaient ou collaient à l'occasion des perles sur leurs armes en une ébauche de marqueterie. Parmi les dix casse-têtes très élaborés portant des décorations de ce type dont nous disposons à ce jour, deux se trouvent à Paris. Fabriqué à partir du nœud de souche d'un érable dans lequel a été taillée la boule, le premier, aujourd'hui conservé à la Bibliothèque Sainte-Geneviève, est décoré selon les principes de la sculpture sur copeaux ${ }^{28}$ et incrusté de perles de wampum (BSG Cat.-no : 113) [fiǵ. 3]. Quinze perles blanches sont incrustées le long du dos de la crosse, la quinzième se trouvant au niveau de la poignée et étant positionnée verticalement de manière à ce que son trou soit visible. Figurent ensuite trois motifs en $\mathrm{X}$ tracés selon la technique de la sculpture en coches
13. Pierre Pouchot (1712-1769) entretint de bonnes relations avec les Premières Nations pendant toute la durée de son mandat de commandant du fort Niagara. Les Senecas, dont les terres abritaient son fort, lui donnèrent le titre honorifique suivant: Sategariouaen/ Sategayogen, ce qui veut dire le «milieu des bonnes affaires». Après la victoire anglaise dans les colonies, on considéra en France qu'il avaitéchouéà mener sa mission à bien. II commença alors à travailler à la publication de son journal de bord, un projet qui ne put aboutir de son vivant car il tomba au combat (Moogk 2003). Tout ceci n'enlève rien à l'intérêt de ses descriptions des coutumes autochtones, qui sont très proches de celles des spécialistes ou de certains de ses contemporains, comme sir William Johnson.

14. Pouchot était très proche des Iroquois de la mission de La Présentation, mais aussi des Mississaugas (Ojibwés de l'est) et d'autres encore (Moogk 2003).

15. II nous manque ce même type d'analyse des couleurs et de leur signification pour les Algonquiens.

16. Les Onnéyotes (aujourd'hui Oneida) font partie des Six Nations iroquoises. Installés à l'ouest de la rivière Mohawk, ils sont parfois désignés comme «gardiens de la porte orientale». Ils se donnaient quant à eux le nom d'Haudenosaunee, «peuple des maisons longues», un nom qui renvoie à leur habitat traditionnel et qui désigne plus largement toutes les nations vivant sur ce territoire

(Fenton 1998: 23, 103 et 221).

17. La même source décrit ce collier comme un «collie français à hachette de très grande taille tissé de 6000 perles wampum»)

(NYCD 1856, t. VII: 358).

18. La meilleure description est celle qu'en fait Bougainville dans une lettre adressée à Marc Pierre de Voyer de Paulmy d'Argenson, ministre des

d'Argenson, ministre des
Affaires étrangères en 1757 :

«Ce collier, suivant les coutumes des nations, appartenait à celle qui avait à l'armée le plus de guerriers. C'était sans contredit aux Iroquois [Mohawks de Kahnawake et de Oka] qui étaient et qui, Presque toujours, sont les plus nombreux et à qui leurs anciennes victoires sur presque toutes les nations de l'Amérique septentrionale ont donné un ton de supériorité qu'ils conservent. ) (NYCD 1858, t. X: 609; Bougainville 2003 [1756-1760]: 213) La traduction anglaise de ces lignes introduit le terme «domiciliated Indians» -domicilié- initialement utilisé par les Français du Canada comme par Beauharnois en 1744: l'expression renvoyait à leurs alliés chrétiens parmi les Amérindiens vivant dans les plaines le long du Saint-Laurent, [...]

(Voir suite des notes p.72) 
[...] comme les Abénakis de Saint-François, les Hurons-Wendat de Lorette, les Mohawks - actuellement Kahnawake et Kanehsatake ainsi que les Micmacs de Restigouche (Phillips 1998 : 83 sqq.; ANOM C11, A 75, fol. 83 sqq.; ANOM C11, A 75, fol.
138-142v. Lettre de Beauharnois au ministre).

19. On connaît très mal le sens et la pratique du scalp chez les Premières Nations d'Amérique du Nord, aussi de nombreuses interprétations erronées circulent-elles. Le travail de James Axtell et William C. Sturtevant peut servir d'introduction générale sur le sujet (1980). Celui de Jean-François Lozier Celui de Jean-François
est plus à jour (2003).

20. En nous reportant à des illustrations de femmes de la période en question, nous constatons que le wampum, qui présente quelques rangs de perles, ressemble à un collier ras-de-cou, comme l'attestent de nombreux documents portant sur les Abénakis etles Pentagouets Abenakis etles Pentagouets et datant d'une époque plus tardive (voir: Tate, cat.- $\mathrm{n}^{\circ}$ : N06243, Portrait de Lady Jane Anstruther par sir Joshua Reynolds, 1761; Bourque et Labar 2009: 24. Stolle 2016: et Labar 2009: 24; Stolle 201 en 1646, on utilisait environ deux mille perles pour ce genre de colliers (Snow et al. [dir.] 1996: 58 sq.), ce qui correspond tout à fait à la description qu'en fait Pouchot.

21. La plupart de ses écrits se basent sur les observations qu'il a pu mener auprès des Iroquois christianisés de La Présentation et de leurs voisins, les Mississaugas (Ojibwés de l'est) [Moogk 2003]

22. Comme nous l'avons déjà mentionné (voir supra, n. 12), les colliers wampum ont partie liée avec le nom des morts. Le guerrier ou le chef qui accepte le collier comme invitation à partir en guerre porte ce nom pendant toute la durée de l'expédition. Pour qu'un captif fût libéré il fallait remettre e collier a quelau'undautre, comme s'il s'agissait d'un lien dont on se libérait. Un document mentionne un prisonnier remis aux Français par les Mohawks en 1646: «Voici [dit le chef] le lien qui le retenait en captivité. prenez le prisonnier et ses prenez le prisonnier et ses
chaînes et faites de lui ce qu'il plaira à Onontio [le gouverneu de la Nouvelle-France].» (Snow etal. [dir.] 1996: 58 sq.) Le témoignage d'Esprit Radisson, capturé par Radisson, capture par les Mohawks avant 1654, confirme cette symbolique: «Mon père chantonne pendant quelques instants, puis prend la parole et défait le collier de perles que je portais au cou avant de le jete aux pieds d'un vieil homme. ll coupe alors la corde qui me retenait prisonnier et $m^{\prime}$ invite à me lever.») (ibid 1996: 80)

(Voir suite des notes p.75) ainsi que, dessous, une seule et unique perle violette. Des marques de piǵments rouges et noirs sont visibles sur la surface très polie de l'objet. Nous ne savons pas à quoi renvoient ces perles.

Le casse-tête a pu faire partie de la collection de Nicolas Fabris de Peiresc (1580-1637), qui aurait été un collectionneur d'objets ethnographiques, mais à ce jour, rien ne prouve cette appartenance. La première trace écrite fiable s'y rapportant est une gravure datant d'avant 1688. Y figure un pan de la collection ethnographique du cabinet de curiosités de la bibliothèque Sainte-Geneviève, mais sans note explicative pour accompaǵner l'image (Du Molinet 1692: s. p.; Zehnacker et Petit 1989: 78; Feest 1992: 78) ${ }^{29}$. La présence d'une perle violette nous aide à dater le casse-tête: en effet, les premières perles violettes auraient fait leur apparition entre 1637 et 1640, ce qui rend peu plausible l'appartenance de l'objet à la collection de Peiresc (Stolle 2016: 251).

Le deuxième casse-tête appartient à la collection du musée du quai Branly - Jacques Chirac (Cat.-n ${ }^{\circ}$ : 71.1917.4.14) [fig. 1, p. 81]. Il est fabriqué dans une seule pièce d'érable, sa boule taillée à partir du nœud de souche. L'arme est bien plus grande et lourde que la précédente et se rapproche davantage du cassetête zoomorphe précédemment décrit Dans l'article de Paz et Niko "Une histoire des wampums..." p. 27. Le dos rectangulaire de la crosse est incrusté de deux rangs de perles alternativement blanches et violettes. D'autres perles de ces deux couleurs forment un motif en $\mathrm{V}$ de part et d'autre de la poignnée de cette arme qui se brandissait à deux mains. Nous ne connaissons pas la signification de ces motifs. Le casse-tête est bien équilibré, ce qui en fait une arme efficace en combat rapproché. Tout comme le précédent, ce maǵnifique objet appartenait à un personnage éminent de sa communauté qui avait les moyens et le droit d'embellir son arme afin qu'elle reflète sa position. En Europe et ailleurs, les armes en métal des aristocrates étaient elles aussi savamment décorées (Breiding 2003).

Concernant cet objet, nous n'avons pas pu remonter avant 1861-1865, moment de son transfert depuis le cabinet des Médailles. Sur la liste des pièces à transférer, il est inclus dans le lot «127-131. Casses-têtes. » (AN F/17/3436, s. p.). Les entrées de l'inventaire de 1865 établi par l'ancien musée de l'Artillerie sont plus précises: elles mentionnent la terre d'oriǵine de ces objets (Afrique, Brésil, Océanie et Amérique du Nord). Le casse-tête numéro 401 est décrit comme suit: «Un casse-tête de l'Amérique du Nord ${ }^{30}$ » (MA inventaire, 1865, s. p.). La forme caractéristique de la pièce, sa poignée à deux mains et l'incrustation de perles de wampum permettent de la dater: il s'agit d'un casse-tête du début XviII siècle, voire plus ancien. Toutefois nous savons très peu de choses sur sa provenance ou l'usage qui en était fait. Là encore, elle pourrait faire l'objet d'un travail de recherche dédié. Des tableaux corroborent la datation : sur la peinture à l'huile que l'on doit à John Verelst intitulée Etow Oh Koam, Roi de la Nation Fleuve, représentant un Mahican, Nicholas de son nom de baptême, ainsi que sur la gravure de 1710 par John Simon, on peut voir un casse-tête comparable en taille et en proportions (fig. 8) [Garratt et Robertson 1985: 7 et 98; Hinderaker 1996: 512) ${ }^{31}$. À droite d'Etow Oh Koam, en arrière-plan, une scène de combat oppose deux guerriers : l'un gît à terre, son casse-tête brandi pour se protéger, tandis que l'autre s'apprête à le frapper du sien (Brasser 1978: 201).

\section{GONGLUSION}

Les wampums étaient manifestement partie intégrante des sociétés amérindiennes, notamment chez les Iroquoiens des terres boisées orientales. Ces wampums en forme de colliers ou précédemment de branches ne servaient pas seulement à donner un nom: autour du cou d'un captif, ils étaient une marque de propriété. Ces colliers avaient également une valeur rituelle puisque ce sont eux qu'on remettait aux guerriers lorsqu'on avait fini de faire ses condoléances. Ils étaient présentés afin de compenser la mort d'un homme ou d'une femme: il s'aǵissait de lier un guerrier ou un chef au collier en question et au nom de son propriétaire, de sorte qu'il revienne avec un prisonnier susceptible de remplacer le disparu au sein de la communauté. À la différence des autres colliers de wampum, ceux-ci étaient peints en rouge, c'est-à-dire badigeonnés de vermillon. Ils étaient également traités différemment: on les jetait par terre et ceux qui les ramassaient s'enǵageaient à participer à l'expédition à venir. Ce traitement peu respectueux allait de pair avec le fardeau, l'immense responsabilité dont ils chargeaient les individus enrôlés. Le rouge souligne cet aspect-là car, chez les Iroquoiens, le rouge est la couleur du sang et de la vie. La couleur renvoie donc à l'obligation de faire des prisonniers afin de compenser la perte subie par la communauté. La taille du collier importait: un petit équivalait à une invitation à participer à une expédition intertribale et un grand à une campagne confédérale. La durée de vie de ces colliers était relativement courte; ils ne survivaient guère à une expédition. Une fois leur tâche accomplie, les nouveaux propriétaires - les guerriers les plus valeureux ou la nation numériquement supérieure - détissaient l'objet pour lui donner une nouvelle fonction et se libérer de leur fardeau. La plupart du temps, ses perles servaient à confectionner des bijoux, des colliers d'alliance (comme les colliers votifs) ou des colliers pour lier communautés autochtones et ordres ou saints catholiques (voir: Havard; Núñez-Regueiro et Stolle dans ce numéro).

L'existence physique de ces colliers liés à la guerre avait beau être courte, ils étaient immortalisés par leurs propriétaires, des guerriers, qui les entaillaient, les gravaient ou les peiǵnaient sur leurs casse-têtes, sur des arbres et sur leurs corps. En effet, ils permettaient d'identifier les chefs de guerre; en tant que symboles hiérarchiques, ils jouaient le rôle des médailles en Europe. Ils étaient également un gage de la parole donnée et avaient pour autres fonctions de représenter le nom d'un mort et les possessions du clan (notamment les prisonniers). En tant que symboles hiérarchiques, c'étaient leur présence et leur accumulation qui 


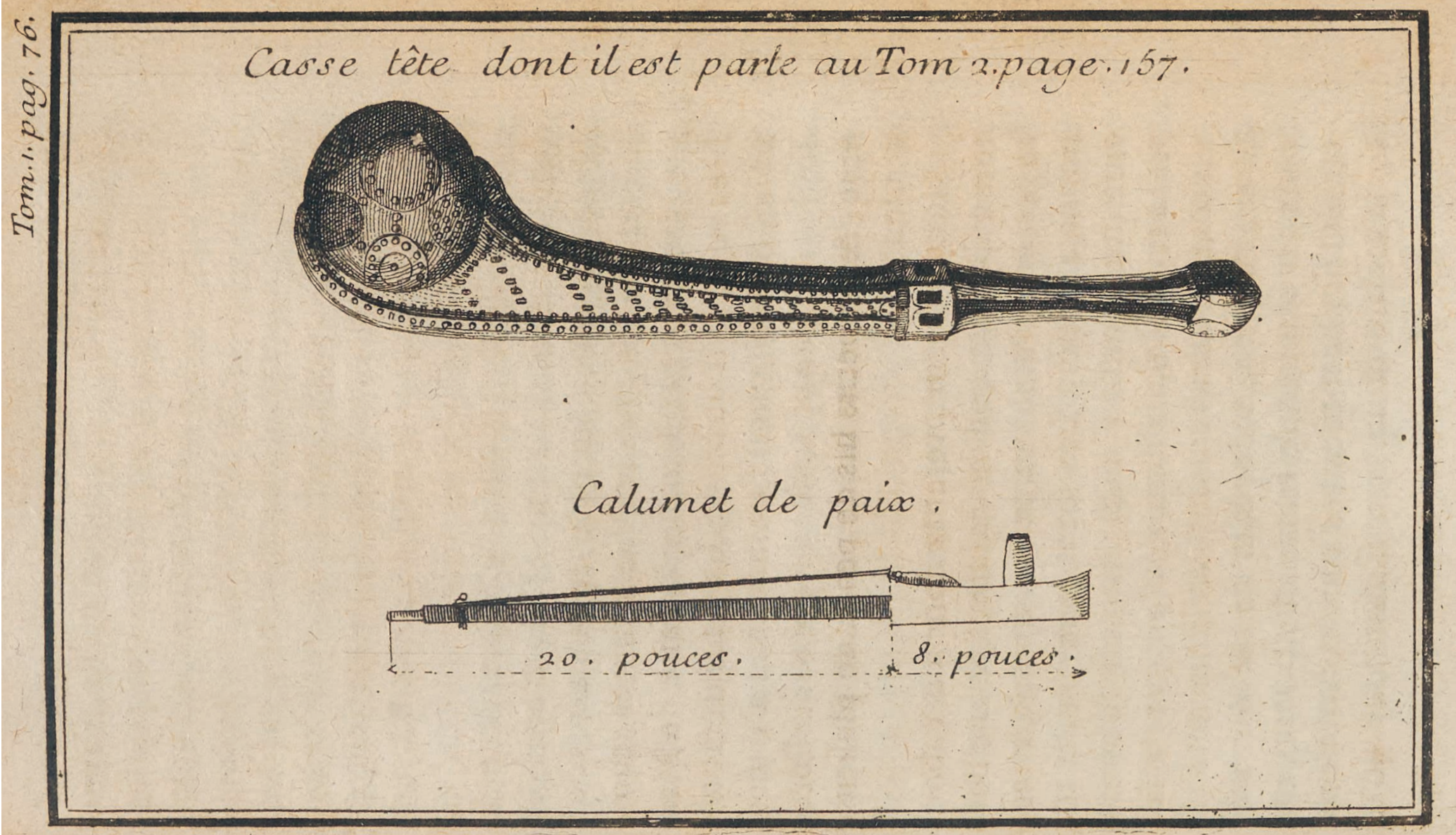

fig. 7. Claude Charles Le Roy de la Potherie, dit Bacqueville de La Potherie, Histoire de l'Amérique septentrionale..., tome 1, imprimé à Rouen, se vend à Paris, 1722. $\mathrm{BnF}$, département Réserve des livres rares, 8-P-340 (1), p. 76 


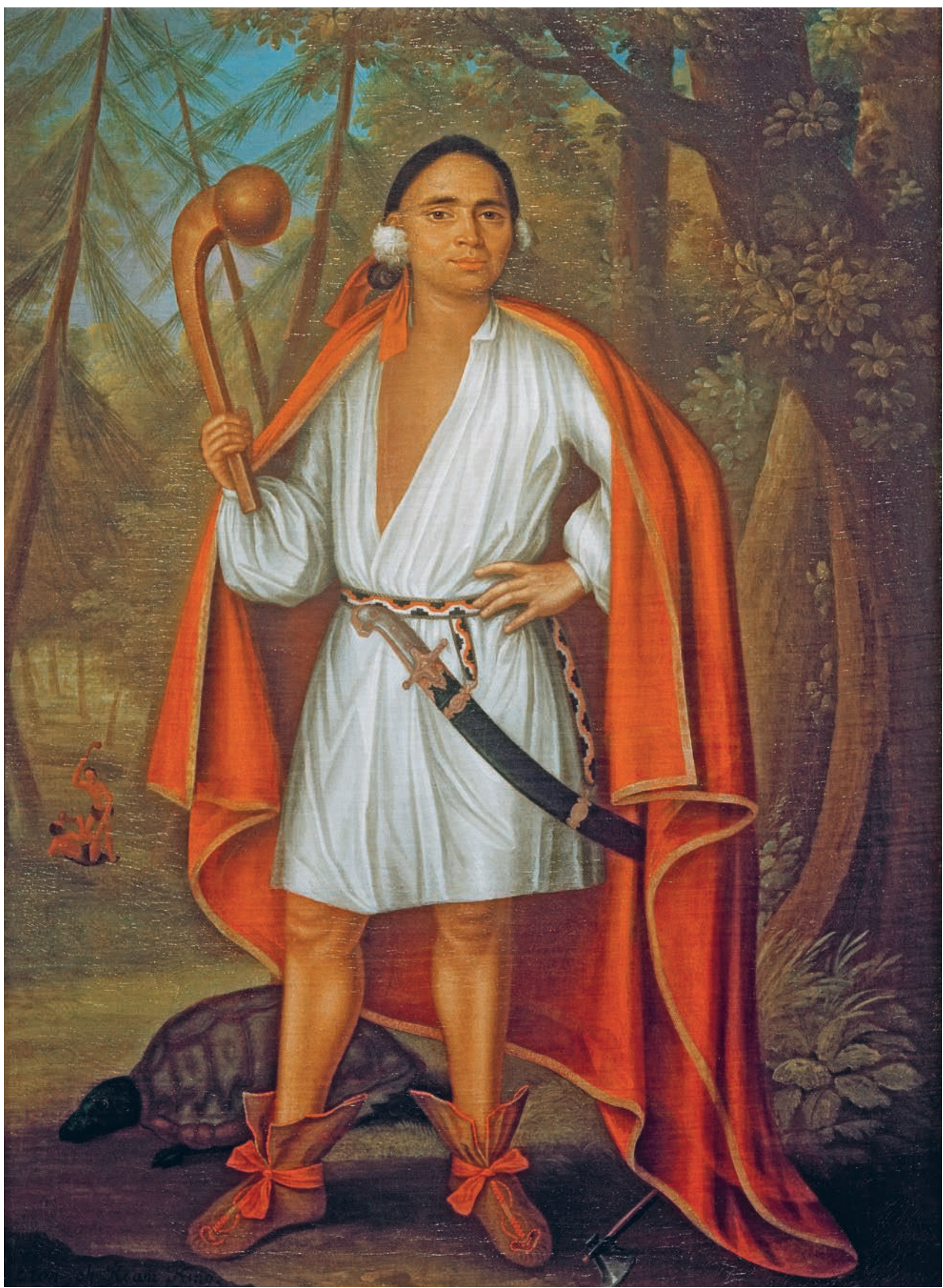

fig. 8. John Verelst, «Etow Oh Koam, Roi de la River Nation», de son nom de baptême Nicholas, un Mahican, 1710. Huile sur toile, $91,5 \times 64,5 \mathrm{~cm}$. Bibliothèque et Archives Canada / Library and Archives Canada, NAC C-092421. 
faisaient foi, comme l'attestent de nombreuses sources graphiques. Nous ne pouvons à ce jour étendre cette symbolique qu'aux casse-têtes décorés de perles. Les perles de wampum étaient précieuses; le fait qu'elles décorent des armes rappelle des pratiques euroaméricaines, arabes ou asiatiques d'incrustation de pierres précieuses dans le noble métal qui servait à forger les armes. Ces décorations précisent le statut du propriétaire de l'arme en question, qui donne à voir son rang en la brandissant. Chez les Amérindiens, les porteurs de ces armes étaient souvent des chefs. Chez les Iroquois, les meilleurs chefs de guerre se voyaient conférer des titres suivant leur mérite plutôt que leur ascendance. Le plus connu d'entre eux est peut-être Thayendanega, "Chef des pins» de son titre, Joseph Brant de son nom de baptême, un Mohawk (1743-1807) à qui l'on faisait don de casse-têtes ou de pipes tomahawk en signe d'amitié. L'un de ces objets porte une plaque en argent sur laquelle on peut lire la phrase suivante: «Offert à mon ami Joseph Brant, de la part du Duc de Northumberland 1805» (Green et Fernandez 1999: 53).

Traduit de l'anǵlais par Lucie Modde

musée du quai Branly-Jacques Chirac Nikolaus.Stolle@quaibranly.fr
23. La première mention écrite d'une cérémonie au cours de laquelle un individu reçoit un nouveau nom date de 1642 , chez les Hurons (les HuronsWendat actuels): «Cette ceremonie se fait en vn festin solemnel en presence de plusieurs conuiez, celuy qui fai reuiure le trepassé; fait vn present à celuy qui doit prendre sa place: il luy met parfois vn collier de Pourcelaine au col.» (JR1898, t. XXII: 288 sq.) Comme c'est encore le cas aujourd'hui, le nom est remis pour un temps limité et reviendr au clan à la mort de l'individu afin qu'il puisse être donné à un autre.

24. À savoir les Mohawks (de Kahnawake, de Kanesatake), les Hurons-Wendat de Wendake, les Abénakis et les Algonquins (Lozier 2012: 176).

25. Il est vraisemblable que ces incisions aient été rehaussées de couleurs, ce que nous indiquent certains spécimens conservés à ce jour Mais à l'exception de quelques rares traces, la peinture a disparu de la plupart des casse-têtes (voir: casse-tête au Musée canadien de I'histoire III-I-1493 datant d'avant 1747; Meachum 2007: 71).

26. Tout comme chez Lafitau, la copie française du dessin seneca fournit la description suivante: «Voila comme ils marquent quand Les collier qui dont Eté donné pour lever un partis de guerre et Venger La mort de quelqûns, Leur apporteneint ou a quil qu'une dela mesme famille.») (Anom C 11, A 4: fol. 268; O'Callaghan 1849: 9)

27. Le casse-tête porte deux marques du musée de l'Artillerie Un poinçon ovale comportant les mots «MUSÉE DE L'ART ${ }^{i e}$ » et le chiffre «1» a été appliqué à deux reprises. Le chiffre renvoie à la salle d'exposition: en effet, les casse-têtes se trouvaient dans les «Galeries $\mathrm{N}^{\circ} 1$ et $\mathrm{N}^{\circ} 2 »$ (Anonyme 1826: 38). Au fil des décennies suivantes, le casse-tête reçut d'autres numéros d'inventaire, dontle dernier, K. 75, par Penguilly en 1862. Ce numéro est encore visible sur une étiquette imprimée et collée à même l'objet. Pour une hypothèse concernant ses origines, voir: Delpuech etal. 2019, dont le travail méticuleux a permis d'établir la provenance d'une pièce ethnographique autochtone originaire d'Amérique du Nord. L'objet, qui date d'avant 1735 , fait partie des collections du prince de Condé, dont l'ancêtre l'avait reçu d'Antoine-Denis Raudot (1679-1737), intendant de la Nouvelle France (1705-1711).
28. Cette technique décorative est typique des Abénakis/Pentagouets et des Passamaquoddys vivant dans le Québec et le Maine actuels, mais aussi des Mahicans avant 1740 (Brasser 1978: 208). L'art de la sculpture en coches a également été observé chez les Senecas avant 1812 (Fenton 1978: 307) et chez les Cayugas avant 1845 (Vennum 2008 [1994]: X sq.). Une recherche approfondie mériterait d'être menée sur le sujet.

29. L'histoire de la collection ethnographique de la bibliothèque Sainte-Geneviève n'est pas bien documentée. Si l'on confronte les objets ethnographiques actuels avec les listes des objets confisqués pendant la Révolution française, on observe que beaucoup de choses ont changé. de choses ont changé. une attribution fautive: I'objet censé être le plus vieux carquois connu des Premières Nations d'Amérique du Nord esten réalité un objet sénégalais datant d'avant1740. Seules

des recherches poussées permettraient d'aboutir à des réponses plus complètes (Zehnacker et Petit 1989: 80).

30. Voir supra, n. 27 Le casse-tête porte le même poinçon ovale mais sans numéro.

31. Le dessin représentant un casse-tête réalisé par Bacqueville de La Potherie en 1722 n'aide pas vraiment à sa contextualisation. En effet, il laisse beaucoup de place à l'imagination: les pièces incrustées pourraient être du verre plutôt que des perles (Bradley et Karklins 2012: $95 \mathrm{sq}$.). La description qui l'accompagne n'est guère plus satisfaisante, à l'exception de cette phrase: «On presenta le casse-tête aux Hurons. [...] Le casse-tête est une maniere de hache-d'arme qui est le simbole d'une guerre que I'on déclare.» (Bacqueville de La Potherie 1722, †. II: 157) Au vu de cette phrase, l'illustration serait plutôt d'origine française qu'autochtone (voir: Feest 1992: 79 sq.).
Remerciements

Nous remercions Christine Duvauchelle et Olivier Renaudeau pour l'aide accordée dans le cadre de cette recherche. 
Bibliographie

SOURCES MANUSCRITES

ANOM (Archives nationales d'outre-mer)

[1666] C 11, A 4 La Nation Iroquois : ANOM C 11, A 75 ,fol. 138-142v. Lettre de Beauharnois au ministre: Lorette et Lac de Deux Montaǵne... 1741, Sept. 21

\section{AN (Archives Nationales,} site Paris)

1793-1794 AN AJ/15/836 Collections de Chantilly : s. p.

1861-1878 AN F/17/3436 Translation d'armes modernes et orientales au musée de l'Artillerie et au musée des Souverains: s. p AN F/17/3978 dos. 3: Relatif au transport des collections de Chantilly au Muséum d'histoire naturelle: s. p.

Bnf (Bibliothèque nationale de France), bibliothèque de l'Arsenal

1744 Ms 3459, Dumont de Montigny, Poème en vers touchants l'établissement de la province de la Louisianne connue sous le nom du Missisipy.

\section{MA (musée de l'Armée)}

1865 Inventaire: s. p.

\section{SOURCES IMPRIMÉES}

\section{Anonyme}

1826 Notice abrégée des collections dont se compose le musée de l'artillerie. Paris, Huzard-Courcier.

\section{Axtell, James}

et Sturtevant, William C. 1980 «The Unkindest Cut, or Who Invented Scalping? », The William and Mary Quarterly 37(3) : 451-472.

\section{Bacqueville de La Potherie, Claude-Charles Le Roy}

1722 Histoire de l'Amérique septentrionale, t. II-III. Paris, Jean-Luc Nion et François Didot.

\section{Bandau, Anja, Dorigny,} Marcel et von Mallinckrodt, Rebekka (dir.)

2010 Les mondes coloniaux à Paris au XVIII siècle: circulation et enchevêtrement des savoirs. Paris, Karthala.

\section{Baugy, Louis-Henri (de)}

1883 Journal d'une expédition contre les Iroquois en 1687. Paris, Ernest Leroux.

\section{Bérenger, Jean-Pierre}

1790 Collection abrégée des voyages faits autour du monde par les différentes nations de l'Europe, t. VI. Paris, Lejay fils.

\section{Bougainville,}

Louis-Antoine (de)

2003 [1756-1760] Écrits sur le Canada: Mémoires, journal, lettres. Sillery, Septentrion.
Bourque, Bruce J. et A. Labar, Laureen

2009 Uncommon Threads:

Wabanaki Textiles, Clothing,

and Costume. Augusta/Seattle/

Londres, Maine State Museum/ University of Washington Press.

Boyer, Abel

1729 The Royal Dictionary, French and English, and

English and French. Londres,

J. \& J. Knapton et al.

\section{Bradley, Charles}

\section{et Karklins, Karlis}

2012 «A Wampum-Inlaid

Musket from the 1690 Phips

Shipwreck», Beads 24: 91-97.

Brasser, Ted J.

1978 «Mahican », in William C. Sturtevant (dir.), Handbook of the North American Indians, t. XV. Washington, Smithsonian Institution : 198-212.

\section{Breiding, Dirk H.}

2003 The Decoration of Arms and Armor. Heilbrunn Timeline of Art History [en ligne], disponible sur: https://www metmuseum.org/toah/hd/ decaa/hd_decaa.htm (consulté le 28 juin 2021)

\section{Callender, Charles}

1978 «Shawnee», in Bruce G. Trigger (dir.), Handbook of the North American Indians, t. XV. Washinǵton, Smithsonian Institution : 622-635.

\section{Clark, Matthew St. Clair} et Lowrie, Walter (éd.) 1832 [1789-1815] American

State Paper: Documents, legislative and executive, of the Congress of the United States, t. IV. Washington, Gales \&

Seaton.

\section{Cotgrave, Randle}

1611 A Dictionary of the French and English Tongres. Londres, Adam Islip.

\section{Delpuech, André, Roux,} Benoît et Saulieu (de), Geoffroy

2019 «Un intendant en quête de curiosités. Les collections natchez de Louisiane du cabinet Raudot. A steward in search of curiosities", in Dominique Barjot et Denis Vialou (dir.), La NouvelleOrléans 1718-2018: regards sur trois siècles d'histoire partagée - Deuxièmes Entretiens d'outre-mer. Paris, Académie des sciences d'outre-mer/Maisonneuve et Larose : 161-174.

\section{Drolet, Gilles}

1989 Missionnaire en Nouvelle-France: PierreJoseph-Marie Chaumonot (1611-1693). Québec, Anne Siǵier.

\section{Du Molinet, Claude}

1692 Le Cabinet de la bibliothèque de Sainte-Geneviève. Paris, Antoine Dezallier

\section{Feest, Christian}

1992 "North America in European Wunderkammer Before 1750 . With a Preliminary Checklist », Archiv für Völkerkunde 46: 61-109.

\section{Fenton, William N.}

1978 «Northern Iroquoian Culture Patterns », in William C. Sturtevant (dir), Handbook of the North American Indians t. XV. Washington, Smithsonian Institution : 296-321. 1998 The Great Law and the Longhouse: A Political History of the Iroquois Confederacy. Norman, University of

Oklahoma Press.

\section{Forster, Michael K.}

1996 «Language and the Culture History of North America », in Ives Goddard (dir.), Handbook of the North American Indians, t. XVII. Washington, Smithsonian Institution : 64-110

\section{Franquet, Louis}

1889 Voyages et Mémoires sur le Canada. Québec, À. Coté et Cie.

\section{Furetière, Antoine}

1690 Dictionaire universel, contenant generalement tous les mots François..., t. II. Le Havre/Rotterdam, Arnout et Reinier Leers.

Garratt, G. John, et Robertson, Bruce

1985 The Four Indian Kings = Les Quatre Rois Indiens. Ottawa, Public Archives of Canada.

\section{Girard, Renée}

2020 (9 novembre) «A root "that our French call rosary": Foodways in Indigenous and French North America », Borealia, Early Canadian History [en liğne], disponible sur: https://earlycanadianhistory.ca/2020/11/09/a-rootthat-our-french-call-rosaryfoodways-in-indigenous-andfrench-north-america/ (consulté le 7/09/2021).

\section{Goddard, Ives}

1978 «Eastern Alǵonquian Lanǵuaǵes », in Bruce G. Trigger (dir.), Handbook of the North American Indian, t. XV. Washington, Smithsonian Institution : 70-77.

\section{Green, Rayna,}

et Fernandez, Melanie

1999 The British Museum Encyclopedia of Native North America. Londres, British Museum Press.

Hamell, George R.

1992 "The Iroquois and the World's Rim: Speculations on Color, and Contact», American Indian Quarterly 16 (4) 451-469.

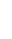

Hamilton, Edward P. (éd.)

Adventures in the wilderness: the American journals of Louis Antoine de Bougainville 1756-1760. Norman, University of Oklahoma Press, 1964.

\section{Harrison, Samuel A. (éd.)}

1876 Memoir of Lieut. Col. Tench Tilghman, secretary and aid to Washington: together with an appendix, containing revolutionary journals and letters, hitherto unpublished. Albany, J. Munsell.

\section{Havard, Gilles}

2007 Empire et métissages: Indiens et Francais dans le Pays d'en Haut, 1660-1715. Sillery, Septentrion.

\section{Hinderaker, Eric}

1996 "The "Four Indian Kings" and the Imaginative Construction of the British Empire », The William and Mary Quarterly 53 (3)

487-526.

JR Thwaites, Reuben G. (éd.) 1896-1901 The Jesuit Relations and Allied Documents: Travels and Explorations of the Jesuit Missionaries in New-France, 1610-1791; the Original French, Latin, and Italian Texts, with English Translations and Notes, t. I-LXXIII. 


\section{Stolle, Nikolaus}

2012 «Geköpft, geschunden und gezeichnet. Zur Darstellun von Schädel- und Kopftrophäen im indigenen, östlichen Nordamerika des 17. bis 19 . Jahrhunderts », in Afried

Wieczorek, Wilfried Rosendah et Andreas Schlothauer (dir.), Der Kult um Kopf und Schödel. Interdisziplinäre Betrachtungen zu einem Menschheitsthe$m a$, actes de colloque.

Mannheim, Reiss-Engelhorn-Museen Mannheim :

78-84

2016 Talking Beads: The History of Wampum as a Value and Knowledoe Bearer, From Its Very First Beginnings Until Today. Hamburg, Dr. Kovač.

2018 «Recorded Honours/ Signs of Conquest: On the Meaning of War Clubs in Indigenous Eastern North America Before 1850 », in Tom Crowley et Andy Mills (dir.), Weapons, Culture and Anthropology Museums. Norwich, Cambridge Scholars Publishing : 66-81.

\section{Vennum, Thomas}

2008 [1994] Lacrosse,

Little Brother of War. Baltimore, The John Hopkins University Press.

\section{Zehnacker, François}

\section{et Petit, Nicolas}

1989 Le Cabinet de curiosités de la bibliothèque Sainte-Geneviève. Paris, Bibliothèque Sainte-Geneviève.

Ci-contre et ouverture

John Verelst, «Etow Oh Koam, Ro de la River Nation», de son nom de baptême Nicholas, un Mahican, 1710.

Huile sur toile, $91,5 \times 64,5 \mathrm{~cm}$ Bibliothèque et Archives Canada Library and Archives Canada, NAC C-092421(détail)

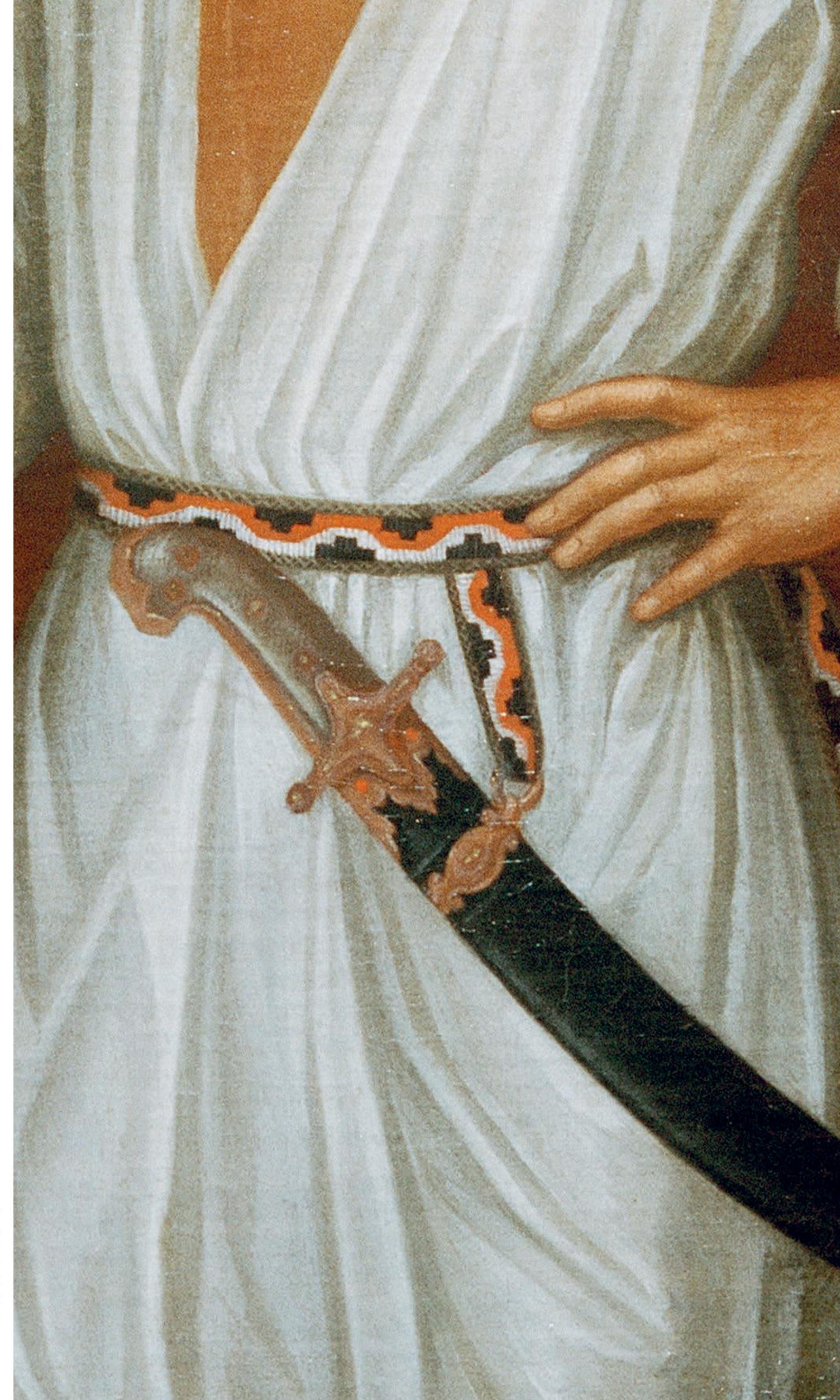

